\title{
GESTIÓN DE TESORERÍA EN HOSPITALES PRIVADOS DEL ESTADO ZULIA, VENEZUELA
}

\author{
Mayerly Parra Fuenmayor \\ https://orcid.org/0000-0003-2197-7446 \\ María Alejandra Ferrer ${ }^{* *}$ \\ https://orcid.org/0000-0001-5401-1838
}

RECIBIDO: Octubre 2019 / ACEPTADO: Enero 2020 / PUBLICADO: Mayo 2020

Como citar: Parra Fuenmayor, Mayerly; Ferrer, María Alejandra. (2020). Gestión de tesorería en hospitales privados del estado Zulia, Venezuela. Telos: revista de Estudios Interdisciplinarios en Ciencias Sociales, 22 (2), Venezuela. (Pp.281-294).

DOI: www.doi.org/10.36390/telos 222.03

\section{RESUMEN}

El objetivo de este trabajo fue analizar la gestión de tesorería en los hospitales privados del municipio Maracaibo del estado Zulia (Venezuela), con base en la gestión del efectivo y la gestión del financiamiento a corto plazo, y sustentada en los aportes de Olsina (2009), Van Horne y Wachowicz (2010), Gitman y Zutter (2012), Ross et al (2013), y Block et al (2013). El estudio se considera de tipo descriptivo, transeccional de campo, con diseño no experimental. La población estuvo conformada por los hospitales privados del municipio Maracaibo del estado Zulia (Venezuela) ubicados en el nivel intermedio (color amarillo), según la Cédula para la Clasificación de Hospitales. Los resultados evidencian que los hospitales privados mantienen saldos de efectivo excedentarios invertidos en valores negociables, manejan fluctuaciones estacionales mediante activos líquidos, controlan la recuperación de cuentas por cobrar y disponen de saldos suficientes para la cancelación de cuentas por pagar; por otro lado, el financiamiento a corto plazo básicamente es destinado a la adquisición de insumos y al pago de proveedores. Se concluyó que los hospitales privados deben fortalecer las políticas y mecanismos para una gestión de tesorería eficiente y eficaz.

Palabras clave: hospitales, gestión de tesorería, efectivo, financiamiento a corto plazo

Treasury management in private hospitals in Maracaibo, Zulia state, Venezuela

\section{ABSTRACT}

The aim of this work was to analyze the treasury management in the private hospitals in Maracaibo (Zulia, Venezuela), considering cash management and short-term financing

\footnotetext{
"Licenciada en Contaduría Pública. Magister en Gerencia de Empresas Mención Gerencia Financiera.

Docente-Investigadora de la Universidad del Zulia. Profesora Titular. Licenciada en Contaduría Pública, Magister en Gerencia de Empresas Mención Gerencia Financiera, Doctora en Finanzas de Empresa (Universidad Complutense de Madrid-UCM). Correo electrónico: maferrer99@yahoo.com
} 
management, and based on the contributions of Olsina (2009), Van Horne and Wachowicz (2010), Gitman and Zutter (2012), Ross et al. (2013), and Block et al. (2013). The study is considered descriptive, field transectional, with non-experimental design. The population was made up of private hospitals in Maracaibo (Zulia, Venezuela) located in the intermediate level (yellow color) according to the classification of hospitals. The results show that private hospitals maintain surplus cash balances invested in marketable securities, handle seasonal fluctuations through liquid assets, control the recovery of accounts receivable and have sufficient balances for the cancellation of accounts payable; on the other hand, short-term financing is basically intended for the acquisition of goods and the payment of suppliers. It is concluded that private hospitals must strengthen the policies and mechanisms for efficient and effective treasury management.

Keywords: hospitals, treasury management, cash management and short-term financing management.

\section{Introducción}

Los hospitales son tipos específicos de organización social, constituidos para proveer servicios a la comunidad. La naturaleza de la actividad hospitalaria comprende las relaciones entre el hospital y su entorno, las cuales se ven afectadas profundamente por el papel de dicha institución en la sociedad.

Ahora bien, el principal problema financiero del sector salud en países con altos niveles de inflación y procesos acelerados de devaluación, además de la cantidad de recursos disponibles, es la orientación y uso de dichos recursos hacia aquellas áreas o servicios con una baja relación costo-beneficio. Esto último, no se refleja en la mejora de las condiciones de salud de la población asistida, de manera que una mayor cantidad de recursos financieros destinados al sector salud no necesariamente garantiza mejoras en el desempeño de las instituciones y en las condiciones de salud de la población.

No debe entenderse la gestión de fondos exclusivamente como cantidad y flujo de recursos financieros, esta incluye aspectos relacionados con procesos administrativos, así como capacidades técnicas. La gestión financiera debe estar enmarcada por las políticas generales y la misión institucional. Su interrelación con el resto de los servicios es múltiple, por lo cual es vital contar con herramientas técnicas para conocer y analizar los componentes involucrados en cada proceso.

En una empresa comercial, los gerentes financieros tienen por objetivo maximizar la riqueza de los accionistas; sin embargo, en una institución de servicios con cierto carácter social, la meta es lograr el mejor uso posible de los recursos en procura de generar un bienestar común (Terrazas Pastor, 2009). Este hecho se constituye en una de las principales razones por las cuales la gestión financiera en los hospitales debe ser integral, involucrando a todos los actores partícipes en el proceso de atención, quienes actúan como gerentes de área y como tales responsables no sólo del resultado clínico sino del financiero.

Hacia el interior, la gestión financiera de los hospitales debe velar porque los recursos económicos sean utilizados de la manera más eficiente; para garantizar la oportunidad y la calidad de los servicios y el cumplimiento de las obligaciones con terceros. Lo anterior debe ir en línea con una estrategia financiera adecuada, pues de lo contrario se evidenciarán problemas 
para cumplir su misión y objetivos estratégicos (Sanz, 2013). De esta manera, el éxito de una buena gestión en los ingresos, gastos, calidad y precios de los equipos, medicamentos, elementos de consumo, y mantenimiento, se verá reflejado en la situación financiera de los hospitales.

Lograr resultados positivos, cuantificables desde el punto de vista médico-asistencial y económico, supone asegurar el funcionamiento adecuado de la institución y sus proyecciones hacia el futuro. Sin embargo, mantener en operaciones a una institución hospitalaria inmersa en procesos inflacionarios y de devaluación requiere esfuerzos importantes en la gestión de los recursos disponibles. La acelerada pérdida del poder adquisitivo de la moneda dificulta hacer frente a los desembolsos en moneda extranjera para la compra de insumos importados, por lo que la prestación del servicio está sujeta a su cancelación en moneda extranjera, o en su defecto, al equivalente en moneda nacional al tipo de cambio actual.

Entre los diferentes procesos propios de la gestión financiera en los hospitales destaca la gestión de tesorería. Esta constituye un área importante al financiar todo el ciclo productivo pues se encarga de administrar y custodiar los recursos financieros, cumpliendo con las necesidades y valores establecidos por los hospitales para la prestación oportuna de los servicios. Si existe una adecuada aplicación de las estrategias de gestión del efectivo, es factible que se presenten problemas de liquidez y se determinen las necesidades de efectivo (García-Aguilar et al, 2017).

El papel de la gestión de tesorería cobra mayor importancia en el caso del sistema institucional de prestación de servicios de salud privada en Venezuela pues este no recibe apoyo financiero del gobierno nacional. Así, los hospitales privados con fines de lucro asumen un papel importante como entes empresariales en la prestación de servicios de salud, por lo que se ven obligados a optimizar los recursos financieros disponibles.

Sin embargo, los hospitales privados no escapan al hecho de que, tal como se mencionó, una mayor cantidad de recursos financieros no necesariamente garantiza mejoras en el desempeño de los hospitales privados. La gestión inadecuada de dichos recursos puede incidir directamente en la atención a cabalidad de las expectativas y demandas de un cliente-el paciente. Por ello, el objetivo de este trabajo fue analizar la gestión de tesorería en los hospitales privados del municipio Maracaibo del estado Zulia, con base en la gestión del efectivo y la gestión del financiamiento a corto plazo.

Desde el punto de vista metodológico, esta investigación es de tipo descriptivo, transeccional de campo, con diseño no experimental. La población estuvo conformada por los cinco (5) hospitales privados que prestan servicio de atención médica del sector salud, ubicados en el nivel intermedio (color amarillo) según la Cédula para la Clasificación de Instituciones de Salud Privadas ${ }^{1}$ que operan en el municipio Maracaibo del estado Zulia (Venezuela) (Tabla 1). Para la recolección de datos se administró un cuestionario autoadministrado conformado por 20 preguntas de selección múltiple aplicado al encargado directamente de la gestión de tesorería de los hospitales privados que conformaron la población (Tabla 1).

\footnotetext{
La cédula fue preparada por el Programa de Administración del Sector Salud y Medicina de Emergencia y Desastre de la Universidad del Zulia (PAS-LUZ)
} 
Tabla 1

Población y Unidades Informantes

\begin{tabular}{|l|c|c|}
\hline \multicolumn{1}{|c|}{ Hospital } & $\begin{array}{c}\text { Gerente de } \\
\text { Finanzas }\end{array}$ & $\begin{array}{c}\text { Jefe de } \\
\text { Tesorería }\end{array}$ \\
\hline Hospitalización Clínico, C.A. & 1 & \\
\hline Centro Médico Docente Paraíso, C.A. & 1 & 1 \\
\hline Policlínica Amado, C.A. & 1 & \\
\hline Hospitalización Falcón, S.A. & 1 & 1 \\
\hline Centro Clínico La Sagrada Familia, C.A. & $\mathbf{4}$ & 1 \\
\hline \multicolumn{1}{|c|}{ Total } &
\end{tabular}

Fuente: elaboración propia.

\section{Consideraciones teóricas sobre la gestión de tesorería}

La tesorería constituye una unidad estratégica que debe manejar en forma efectiva y eficiente los recursos financieros de una empresa (Atencio Finol, 2015), por lo que su gestión establece la primera medida de control de las inversiones en activo corriente. Implica un control de los flujos monetarios y su conexión con los flujos comerciales, es decir, un control del dinero que entra y sale, a dónde va, en qué momento y qué costos, o beneficios, producen esos movimientos.

Básicamente, los objetivos de la gestión de tesorería se centran en asegurar la liquidez de la empresa para atender las obligaciones monetarias, mediante la reducción de las necesidades de financiamiento, así como de los costos financieros y de las transacciones (Olsina, 2009; Promove Consultoría e Información SLNE, 2012), y al mismo tiempo maximizar la liquidez de los fondos retenidos en activo corriente (Promove Consultoría e Información SLNE, 2012). Lo anterior, supone reducir el volumen del efectivo utilizado con el fin de incrementar la rentabilidad, pero sin disminuir el nivel de actividad ni exponer a la empresa a riesgos indebidos en relación con el cumplimiento de sus obligaciones financieras.

\section{Gestión del efectivo}

La gestión de efectivo es una actividad mucho más compleja que la simple cancelación de deudas y recepción de pagos a cambio de bienes y servicios, supone mantener un nivel de liquidez adecuado y cumplir con las obligaciones de pago. Según Block et al., (2013), la gestión del efectivo consiste en ejercer control sobre las entradas y salidas de efectivo con la finalidad de minimizar los saldos de efectivo improductivos, a fin de mantener una cantidad de fondos adecuada para poder realizar transacciones y mantener saldos de compensación. Esto es, sincronizar los flujos de entrada y salida para cumplir satisfactoriamente con el ciclo operativo (Albornoz, 2008).

Con relación a los saldos de efectivo, las empresas mantienen dichos saldos por varias razones (Keynes, 1936): motivos transaccionales, motivos preventivos y motivos especulativos. Los saldos de efectivo por motivos transaccionales permiten satisfacer las necesidades de efectivo asociadas al curso normal de las operaciones de la empresa. (Van Horne y Wachowicz, 2010; Ross et al, 2013), considerando la asincronía entre las entradas y salidas de efectivo en las operaciones diarias, por lo que el monto requerido varía de una empresa a otra.

Los saldos de efectivo por motivos preventivos son necesarios para satisfacer necesidades 
inesperadas de efectivo (Van Horne y Wachowicz, 2010; Ross et al, 2013). Se busca disponer de saldos de efectivo para satisfacer necesidades futuras, entre las que se encuentran los desembolsos fijos programados para fechas específicas, como pagos de dividendos e impuestos, erogaciones de capital y re-pagos de préstamos o emisiones de bonos. En contrapartida, una empresa puede disponer de efectivo por nuevas ofertas de obligaciones 0 acciones antes de emplear esos fondos con propósitos de expansión.

Por último, es común que las empresas mantengan saldos de efectivo por motivos especulativos. La acumulación de grandes saldos de efectivo les permite afrontar demandas extraordinarias de efectivo y/o aprovechar oportunidades de inversión a corto plazo (Van Horne y Wachowicz, 2010), con tasas de interés atractivas (Ross et al, 2013), así como una baja súbita en el precio de los productos (Van Horne y Wachowicz, 2010).

Adicionalmente, las empresas deben conservar saldos de efectivo en cuentas bancarias; esto es, mantener saldo de compensación (Gitman y Zutter, 2012; Ross et al, 2013). Este saldo permite a las empresas compensar los créditos obtenidos y/o los servicios del banco o bancos de los que son clientes.

Ahora bien, una adecuada gestión del efectivo descansa en el control de entradas y salidas de efectivo eficiente (Van Horne y Wachowicz, 2010). Ello se basa en las políticas diseñadas para agilizar los cobros y retrasar los pagos (Gitman y Zutter, 2012), de manera que contribuyen al incremento del saldo de efectivo de una empresa y el proporcionan fondos que se puede utilizar en otras inversiones redituables.

La agilización de las entradas de efectivo permite disponer del mismo a la mayor brevedad (Van Horne y Wachowicz, 2010). Supone recuperar las cuentas pendientes lo más pronto posible sin perder ventas a causa de técnicas de cobranza sofocantes (Gitman y Zutter, 2012); estas incluyen los descuentos por pronto pago económicamente favorables. Adicionalmente, se ha de considerar la posibilidad de cobros centralizados o descentralizados, considerando que el primero supone la concentración de los cobros minimizando las demoras en la recuperación de cuentas. Por otra parte, el aplazamiento de los pagos supone la cancelación de las cuentas pendientes lo más tarde posible, sin dañar por ello la reputación crediticia de la compañía, aprovechando cualquier descuento en efectivo que resulte favorable (Gitman y Zutter, 2012).

Cuando la acumulación de reservas de efectivo excede las necesidades diarias, dichas reservas pueden ser invertidas en valores negociables, considerando factores como intereses a devengar, costos de la transacción y variabilidad de flujos de efectivo de la empresa. Los valores negociables son instrumentos del mercado de dinero a corto plazo, que devengan intereses y pueden convertirse con facilidad en efectivo (Gitman y Zutter, 2012).

La selección de los valores negociables se basa en (Olsina, 2009; Van Horne y Wachowicz, 2010; Ross et al, 2013): la seguridad o riesgo de incumplimiento, probabilidad de recuperar el capital invertido inicialmente; comerciabilidad o bursatilidad, habilidad para vender el título en un período corto de tiempo sin pérdida significativa en el precio; vencimiento de los valores negociables; y rendimiento, beneficio (interés), obtenido por el capital invertido. Si bien, la decisión sobre en qué valores negociables invertir se basa en un análisis conjunto de los criterios mencionados, ha de tomarse en cuenta el tratamiento fiscal, entre otros aspectos colaterales (Iturralde Jainaga et al, 2005; Olsina, 2009). 


\section{Gestión del financiamiento a corto plazo}

En términos generales, el financiamiento las empresas permite mantener una economía estable, planear a futuro y expandirse. Así, el financiamiento supone la obtención de recursos monetarios destinados a un fin específico: adquirir bienes y servicios, pagar proveedores, entre otros. Particularmente, el financiamiento a corto plazo supone obligaciones con vencimiento menor a un año, es decir, deben reembolsarse en el plazo de un año, y que son necesarios para sostener gran parte de los activos corrientes de la empresa.

El financiamiento corto plazo asegura la existencia de efectivo para poder evitar su escasez temporal. Es especialmente necesario si la actividad económica se desenvuelve de manera estacional; es inevitable que durante una parte del año los recursos financieros de la empresa se vean sometidos a tensiones; en caso contrario, es posible que sea el resultado del uso ineficaz del efectivo.

Básicamente, las principales fuentes de financiamiento a corto plazo incluyen crédito comercial, préstamos bancarios, papeles comerciales, financiamiento mediante cuentas por cobrar, y financiamiento mediante inventarios.

El crédito comercial o cuentas por pagar constituyen la fuente principal de financiamiento a corto plazo sin garantía (Gitman y Zutter, 2012); es una fuente espontánea de financiamiento en tanto que surge de las operacionales normales de empresa (Block et al, 2013): a medida que aumentan las ventas también lo hace el crédito comercial y se contrae de manera similar cuando las actividades comerciales disminuyen.

Por su parte, los préstamos bancarios se destinan al financiamiento de las necesidades estacionales, la expansión de las líneas de productos y el crecimiento a largo plazo de una empresa (Block et al, 2013). Lo anterior supone un aumento del inventario y de las cuentas por cobrar, los cuales, a medida que se convierten en efectivo, se generan los fondos necesarios para reembolsar estos préstamos (Gitman y Zutter, 2012).

En el caso de economías inmersas en procesos inflacionarios, prevalece el crédito comercial sobre el préstamo bancario, debido a que los proveedores son más abiertos a concederlos y las instituciones bancarias mantienen elevadas tasas de intereses (Torres et al, 2017). Ahora bien, ante tasas de intereses fijas, el costo de financiamiento se diluye en el tiempo haciendo atractivo la obtención de préstamos para la compra de moneda extranjera.

El papel comercial es una forma de financiamiento que consiste en un pagaré negociable a corto plazo, no garantizado (Gitman y Zutter, 2012; Block et al, 2013), que se vende en el mercado de dinero (Van Horne y Wachowicz, 2010). Son emitidos por empresas con una alta capacidad crediticia, generalmente empresas muy grandes, con una solidez financiera incuestionable (Gitman y Zutter, 2012).

El financiamiento mediante cuentas por cobrar exige la pignoración de las cuentas por cobrar como garantía colateral de un préstamo o su venta directa (factoring) (Gitman y Zutter, 2012; Block et al, 2013). En ambos casos, la liquidez de las cuentas por cobrar resulta una forma atractiva de colateral de préstamos a corto plazo (Gitman y Zutter, 2012).

El financiamiento mediante inventario es un convenio de financiamiento basado en el valor de mercado del mismo que es mayor a su valor en libros (Gitman y Zutter, 2012). También han de considerarse la comerciabilidad de los bienes pignorados, la estabilidad de los precios asociados a ellos y la perecibilidad de los productos que lo conforman (Block et al, 2013). 


\section{Gestión de tesorería en hospitales privados}

Considerando la demanda de necesidades de atención de salud, los centros hospitalarios han de optimizar su oferta, tomando en cuenta las exigencias de desembolsos en moneda de extranjera para la cancelación de insumos importados. Para ello, ha de apoyarse en una gestión objetiva y eficiente para la adecuada planificación, organización y control de los recursos disponibles. Así, la gestión de tesorería constituye un elemento clave.

Una de las funciones básicas a considerar con relación a la gestión de tesorería es la gestión del efectivo. Ella involucra tres aspectos fundamentales: mantenimiento de saldos de efectivo, control de entradas y salidas de efectivo e inversiones en valores negociables.

Así, un $60 \%$ de los hospitales consultados dispone de saldos excedentarios de efectivo (Tabla 2), bien por motivos transaccionales, para atender las necesidades diarias, o por motivos preventivos, necesarios para satisfacer las necesidades inesperadas de efectivo con un margen de seguridad (Berk et al, 2010). Un $40 \%$ de los establecimientos hospitalarios afirma disponer siempre de efectivo para manejar las fluctuaciones estacionales en sus flujos de efectivo y un $20 \%$ en algunas ocasiones (Tabla 2); esto les permite afrontar variaciones regulares y predecibles en la actividad económica.

\section{Tabla 2}

Motivos para mantener saldos de efectivo en los hospitales privados del municipio Maracaibo del estado Zulia

\begin{tabular}{|c|c|c|}
\hline Disponibilidad de saldos excedentarios & $\mathrm{Fa}$ & $F r$ \\
\hline Siempre & 1 & $20 \%$ \\
\hline Casi siempre & 1 & $20 \%$ \\
\hline A veces & 1 & $20 \%$ \\
\hline Casi nunca & 1 & $20 \%$ \\
\hline Nunca & 1 & $20 \%$ \\
\hline TOTAL & 5 & $100 \%$ \\
\hline Motivos para mantener saldos de efectivo & Fa & $F r$ \\
\hline Motivos transaccionales & 2 & $50 \%$ \\
\hline Motivos preventivos & 2 & $50 \%$ \\
\hline \multicolumn{3}{|l|}{ Motivos especulativos } \\
\hline \multicolumn{3}{|l|}{ Saldo de compensación } \\
\hline \multicolumn{3}{|l|}{ Otro / Ninguna } \\
\hline TOTAL & 4 & $100 \%$ \\
\hline $\begin{array}{l}\text { Disponibilidad de efectivo para el manejo de fluctuaciones } \\
\text { estacionales de flujos de efectivo }\end{array}$ & $\mathrm{Fa}$ & $\mathrm{Fr}$ \\
\hline Siempre & 2 & $40 \%$ \\
\hline \multicolumn{3}{|l|}{ Casi siempre } \\
\hline A veces & 1 & $20 \%$ \\
\hline \multicolumn{3}{|l|}{ Casi nunca } \\
\hline Nunca & 2 & $40 \%$ \\
\hline TOTAL & 5 & $100 \%$ \\
\hline
\end{tabular}

Fa: Frecuencia absoluta; Fr: Frecuencia relativa

Fuente: elaboración propia, a partir de cuestionario aplicado a los encargados de la unidad de tesorería de cinco (5) hospitales privados del municipio Maracaibo del estado Zulia. 
Las entradas de efectivo de los hospitales consultados provienen en un $60 \%$ tanto de la prestación de servicios como de la recuperación de las cuentas por cobrar generadas por los convenidos con las entidades aseguradoras (Tabla 3), en un $20 \%$ de los casos las entradas de efectivo provienen únicamente de la prestación de servicios y el restante $20 \%$ dispone de efectivo solo mediante la recuperación de las cuentas por cobrar. La diversificación en cuanto a la frecuencia en la percepción de entradas de efectivo dinamiza los flujos en términos del binomio dinero-tiempo, según sostienen Blanch et al (2001).

\section{Tabla 3}

Control de entradas de efectivo en los

hospitales privados del municipio Maracaibo del estado Zulia

\begin{tabular}{|c|c|c|}
\hline Origen de las entradas de efectivo & $\mathrm{Fa}$ & $\mathrm{Fr}$ \\
\hline Prestación de servicios & 1 & $20 \%$ \\
\hline Cobranza & 1 & $20 \%$ \\
\hline Prestación de servicios / Cobranza & 3 & $60 \%$ \\
\hline TOTAL & 5 & $100 \%$ \\
\hline Sistema de cobro en efectivo & $\mathrm{Fa}$ & $F r$ \\
\hline Centralizado & 4 & $80 \%$ \\
\hline Descentralizado & 1 & $20 \%$ \\
\hline TOTAL & 5 & $100 \%$ \\
\hline Recuperación oportuna de cuentas por cobrar & $\mathrm{Fa}$ & $F r$ \\
\hline Siempre & 4 & $80 \%$ \\
\hline \multicolumn{3}{|l|}{ Casi siempre } \\
\hline A veces & 1 & $20 \%$ \\
\hline \multicolumn{3}{|l|}{ Casi nunca } \\
\hline Nunca & & \\
\hline TOTAL & 5 & $100 \%$ \\
\hline
\end{tabular}

Fa: Frecuencia absoluta; Fr: Frecuencia relativa

Fuente: elaboración propia, a partir de cuestionario aplicado a los encargados de la unidad de tesorería de cinco (5) hospitales privados del municipio Maracaibo del estado Zulia

Con relación a la recuperación de las cuentas por cobrar, los resultados muestran que un $80 \%$ de los hospitales cuenta con un sistema de cobro centralizado (Tabla 3), ello evidencia que la mayoría de hospitales cuenta con un sistema ágil e integrado capaz de asegurar un proceso de cobro en tiempo prácticamente real. Ahora bien, indistintamente del sistema de cobro, la totalidad de los establecimientos hospitalarios afirma mantener un control adecuado sobre la recuperación de las cuentas por cobrar (Tabla 3). De esta manera, logran el seguimiento y registro sistemático de los servicios prestados a crédito para su recuperación en el tiempo oportuno.

En los hospitales consultados las salidas de efectivo corresponden en un $60 \%$ a pagos a proveedores, al personal, a organismos públicos y a entidades financieras (Tabla 4). 
Básicamente las salidas de efectivo se realizan mediante un sistema de pago centralizado (Tabla 4), pues ello evita el traslado de fondos, facilita las provisiones de tesorería y asegura la uniformidad de condiciones y sistemas, tal como lo plantean Blanch et al (2001).

Tabla 4

Control de salidas de efectivo en los hospitales privados del municipio Maracaibo del estado Zulia

\begin{tabular}{|l|c|c|}
\hline Principales salidas de efectivo & Fa & Fr \\
\hline Pagos a proveedores & & \\
\hline Pagos al personal & & \\
\hline Pagos a organismos públicos & & \\
\hline Pagos a entidades financieras & 2 & $40 \%$ \\
\hline Otros TOTAL & 3 & $60 \%$ \\
\hline Todas las anteriores TOTAL & $\mathbf{5}$ & $\mathbf{1 0 0 \%}$ \\
\hline \multicolumn{1}{|c|}{ Fa } & Fr \\
\hline Sistema de pagos & 4 & $80 \%$ \\
\hline Centralizado & 1 & $20 \%$ \\
\hline Descentralizado fondos para cancelar cuentas & $\mathbf{5}$ & $\mathbf{1 0 0} \%$ \\
\hline $\begin{array}{l}\text { Control de la disponibilidad de } \\
\text { por pagar }\end{array}$ & $\mathbf{F a}$ \\
\hline Siempre & 5 & $100 \%$ \\
\hline Casi siempre & & \\
\hline A veces & & \\
\hline Casi nunca & & \\
\hline Nunca & $\mathbf{5}$ & $\mathbf{1 0 0 \%}$ \\
\hline
\end{tabular}

Fa: Frecuencia absoluta; Fr: Frecuencia relativa

Fuente: elaboración propia, a partir de cuestionario aplicado a los encargados de la unidad de tesorería de cinco (5) hospitales privados del municipio Maracaibo del estado Zulia.

Las medidas adoptadas por el $100 \%$ de los hospitales les permiten controlar la disponibilidad de fondos para cancelar las cuentas por pagar (Tabla 4). En línea con las ideas de Gitman y Zutter (2012), la gestión del efectivo en los hospitales privados con respecto a las cuentas por pagar no se limita tan solo a la cancelación de sus deudas lo más tarde posible, sino también a controlar la disponibilidad de los fondos tanto para proveedores como para los empleados una vez que los pagos han sido emitidos, es decir, maximizando el tiempo que requieren los desembolsos.

En correspondencia con los bajos niveles de efectivo, un $60 \%$ de los hospitales no invirtió en valores negociables a corto plazo (Tabla 5). Sin embargo, a pesar de los flujos de efectivo escasos, el $40 \%$ restante casi siempre lo hace a través de dichos instrumentos. Con respecto a los determinantes del nivel de efectivo disponible para invertir en valores, un $40 \%$ de los 
hospitales consideró como relevante los costos de transacción relacionados con la compra y venta de valores negociables, para un $20 \%$ es prioritaria la variabilidad de los flujos de efectivo, mientras que un $40 \%$ manifestó no considerar ningún factor para determinar el nivel de efectivo que deben invertir en valores negociables (Tabla 5).

\section{Tabla 5}

Inversiones en valores negociables en los hospitales privados del municipio Maracaibo del estado Zulia

\begin{tabular}{|l|c|c|}
\hline Inversiones en valores negociables a corto plazo & Fa & Fr \\
\hline Siempre & & \\
\hline Casi siempre & 2 & $40 \%$ \\
\hline A veces & & \\
\hline Casi nunca & & \\
\hline Nunca & 3 & $60 \%$ \\
\hline \multicolumn{1}{|c|}{ TOTAL } & 5 & $100 \%$ \\
\hline Determinantes de los niveles de efectivo a invertir en títulos valores & $F a$ & $F r$ \\
\hline Intereses que se obtendrán en un periodo esperado & & \\
\hline Costos de transacción debido a la compra y venta de valores & 2 & $40 \%$ \\
\hline Variabilidad de los flujos de efectivo & 1 & $20 \%$ \\
\hline Otro / Ninguno TOTAL & 2 & $40 \%$ \\
\hline Criterios para seleccionar instrumentos de inversión & 5 & $100 \%$ \\
\hline Seguridad o riesgo de incumplimiento & $F a$ & $F r$ \\
\hline Comerciabilidad o bursatilidad & 2 & $40 \%$ \\
\hline Vencimiento & 1 & $20 \%$ \\
\hline Rendimiento & & \\
\hline Otro / Ninguno & 2 & $40 \%$ \\
\hline TOTAL & 5 & $100 \%$ \\
\hline
\end{tabular}

Fa: Frecuencia absoluta; Fr: Frecuencia relativa

Fuente: elaboración propia, a partir de cuestionario aplicado a los encargados de la unidad de tesorería de cinco (5) hospitales privados del municipio Maracaibo del estado Zulia

Ahora bien, una vez tomada la decisión de inversión, un $40 \%$ de las instituciones hospitalarias considera el riesgo de incumplimiento como criterio para la selección de instrumentos de inversión y un $20 \%$ estudia la bursatilidad del instrumento, frente a un $40 \%$ que no sea plantea criterios de decisión (Tabla 5). De esta manera, el riesgo de incumplimiento del pago del principal o de los intereses constituye el aspecto más relevante para las decisiones de inversión.

Otra de las funciones a considerar con relación a la gestión de tesorería es la gestión del financiamiento a corto plazo. Ella implica la obtención de financiamiento a corto plazo, así como la identificación de las fuentes de financiamiento a corto plazo (Tabla 6). 


\section{Tabla 6}

Obtención de financiamiento a corto plazo en los

hospitales privados del municipio Maracaibo del estado Zulia

\begin{tabular}{|c|c|c|}
\hline Frecuencia del uso del financiamiento a corto plazo & $\mathrm{Fa}$ & $\mathrm{Fr}$ \\
\hline Siempre & 2 & $40 \%$ \\
\hline \multicolumn{3}{|l|}{ Casi siempre } \\
\hline A veces & 2 & $40 \%$ \\
\hline Casi nunca & 1 & $20 \%$ \\
\hline \multicolumn{3}{|l|}{ Nunca } \\
\hline TOTAL & 5 & $100 \%$ \\
\hline Destino del financiamiento a corto plazo & $\mathrm{Fa}$ & $\mathrm{Fr}$ \\
\hline Adquisición de insumos / bienes & 4 & $80 \%$ \\
\hline \multicolumn{3}{|l|}{ Adquisición de servicios } \\
\hline \multicolumn{3}{|l|}{ Pago a proveedores } \\
\hline \multicolumn{3}{|l|}{ Otro } \\
\hline Adquisición de bienes / Pago a proveedores & 1 & $20 \%$ \\
\hline TOTAL & 5 & $100 \%$ \\
\hline $\begin{array}{l}\text { Medios para el financiamiento de las necesidades de } \\
\text { efectivo }\end{array}$ & $\mathrm{Fa}$ & Fr \\
\hline Línea de crédito & 2 & $40 \%$ \\
\hline Banco & 2 & $40 \%$ \\
\hline Otro / Ninguno & 1 & $20 \%$ \\
\hline TOTAL & 5 & $100 \%$ \\
\hline
\end{tabular}

Fa: Frecuencia absoluta; Fr: Frecuencia relativa

Fuente: elaboración propia, a partir de cuestionario aplicado a los encargados de la unidad de tesorería de cinco (5) hospitales privados del municipio Maracaibo del estado Zulia.

Con relación a la obtención de financiamiento a corto plazo, un $40 \%$ de las organizaciones siempre acude al financiamiento a corto plazo y un $40 \%$ solo en ocasiones, en contraposición a un $20 \%$ que manifestó casi nunca hacerlo (Tabla 6). Este tipo de financiamiento permite a las instituciones hospitalarias mantener una economía estable, planear a futuro y asegurar la existencia de efectivo para así evitar periodos.

Se evidenció que un $80 \%$ de los centros hospitalarios destina el financiamiento a corto plazo a la adquisición de insumos/bienes, aunque un $20 \%$ de las entidades encuestadas lo destina tanto a la adquisición de bienes como al pago a proveedores (Tabla 6). De esta manera, el financiamiento a corto plazo permite a las instituciones hospitalarias el desarrollo de sus operaciones para el logro de los objetivos organizacionales planteados, dado el uso eficiente de los flujos de efectivo.

Según los entrevistados, el financiamiento adecuado para cubrir las necesidades de efectivo a corto plazo proviene en un $40 \%$ de los casos de una línea de crédito, en otro $40 \%$ de entidades bancarias, y el $20 \%$ restante no contestó (Tabla 6). De esta manera el acceso a diferentes medios de financiamiento permite a los centros hospitalarios disponer de recursos de efectivo para poder afrontar periodos de escasez temporal de efectivo. 
Si bien un $40 \%$ de los hospitales encuestados, obtiene financiamiento a corto plazo sin garantía, para un $60 \%$ de los casos el financiamiento a corto plazo con garantía constituye la principal fuente de recursos (Tabla 7). Así, agotadas las fuentes de financiamiento a corto plazo sin garantía, las instituciones hospitalarias acuden al financiamiento con garantía al disponer de activos específicos como colateral.

\section{Tabla 7}

Fuentes de financiamiento a corto plazo en los

hospitales privados del municipio Maracaibo del estado Zulia

\begin{tabular}{|c|c|c|}
\hline Tipo de fuente de financiamiento a corto plazo & $\mathrm{Fa}$ & $F r$ \\
\hline Financiamiento sin garantía & 2 & $40 \%$ \\
\hline Financiamiento con garantía & 3 & $60 \%$ \\
\hline TOTAL & 5 & $100 \%$ \\
\hline Tipo de fuente de financiamiento sin garantía & $\mathrm{Fa}$ & $F r$ \\
\hline Crédito comercial & 1 & $20 \%$ \\
\hline Préstamos bancarios & 3 & $60 \%$ \\
\hline \multicolumn{3}{|l|}{ Papeles comerciales } \\
\hline Otro / Ninguno & 1 & $20 \%$ \\
\hline TOTAL & 5 & $100 \%$ \\
\hline Tipo de fuente de financiamiento con garantía & $\mathrm{Fa}$ & $F r$ \\
\hline Financiamiento mediante cuentas por cobrar & 2 & $40 \%$ \\
\hline Financiamiento mediante inventario & 2 & $40 \%$ \\
\hline Otro / Ninguno & 1 & $20 \%$ \\
\hline TOTAL & 5 & $100 \%$ \\
\hline
\end{tabular}

Fa: Frecuencia absoluta; Fr: Frecuencia relativa

Fuente: elaboración propia, a partir de cuestionario aplicado a los encargados de la unidad de tesorería de cinco (5) hospitales privados del municipio Maracaibo del estado Zulia.

En el caso de las fuentes de financiamiento a corto plazo sin garantía, en un $60 \%$ de instituciones hospitalarias estos provienen de préstamos bancarios, mientras que un $20 \%$ de los hospitales acude al crédito comercial (Tabla 7). Lo anterior pone en evidencia el papel de los préstamos bancarios en la gestión de tesorería por constituir la principal fuente de financiamiento para los hospitales privados encuestados. De esta manera, el crédito comercial queda desplazado a un segundo lugar a pesar de ser considerado como la principal fuente de financiamiento a corto plazo.

Adicional a las fuentes de financiamiento sin garantías, un $40 \%$ de los centros hospitalarios recurren al financiamiento mediante cuentas por cobrar, y otro $40 \%$ al financiamiento mediante inventario, al ofrecer los mismos como garantía de crédito (Tabla 7). Este tipo de financiamiento implica la cesión de dichos activos a corto plazo para completar los recursos propios necesarios para sus operaciones.

\section{Conclusiones}

Si bien los hospitales privados buscan ser económicamente rentables, la calidad de los 
servicios que prestan, así como el desarrollo profesional de quienes interactúan en ellos, es primordial. Lo anterior se ve limitado cuando el entorno de dichas instituciones se caracteriza por altos niveles de inflación y procesos acelerados de devaluación. Estos factores condicionan y/o limitan no solo la calidad y oportunidad en la prestación de servicios, sino también una gestión financiera óptima.

De manera particular, la gestión de tesorería vela porque los recursos económicos sean utilizados de la manera más eficiente posible para cumplir con el ciclo operativo. Así, y a pesar del escenario económico descrito, los hospitales privados del municipio Maracaibo del estado Zulia (Venezuela), disponen de saldos excedentarios de efectivo para dar respuesta a las necesidades de efectivo en el curso normal de sus operaciones, así como para desembolsos futuros previamente programados.

La generación de efectivo proviene tanto de la prestación de servicios, como de la recuperación oportuna de las cuentas por cobrar generadas por convenios con entidades aseguradoras. El control de la disponibilidad de fondos por parte de los encargados de la unidad de tesorería de los hospitales privados permite la cancelación de sus compromisos con proveedores, talento humano, organismos públicos y entidades financieras. Adicionalmente, es posible la inversión en títulos valores considerando aspectos como la variabilidad de los flujos de efectivo y los costos de transacción generados por la compra y venta de dichos instrumentos de inversión.

La disponibilidad de efectivo no exime a las instituciones hospitalarias de requerir financiamiento a corto plazo para el cumplimiento de sus operaciones, particularmente para la adquisición de insumos y el pago a proveedores. La obtención de fondos se centra en las oportunidades que ofrecen las entidades bancarias para solventar las necesidades de efectivo. Sin embargo, recientemente el otorgamiento de crédito por parte de la banca ha disminuido significativamente, y en algunos casos, es inexistente, de manera que se requiere el fortalecimiento de políticas y mecanismos para mantener los niveles adecuados de efectivo considerando el ritmo de operaciones de los hospitales.

\section{Referencias bibliográficas}

Albornoz, César (2008) Administración de capital de trabajo: un enfoque imprescindible para las empresas en el contexto actual. Poliantea. Volumen 7. $N^{\circ} 4$. Colombia (pp. 27-51)

Atencio Finol, Aneida (2015). La Tesorería como Unidad Estratégica en la Gerencia Actual.

Revista Venezolana de Gerencia. Volumen 20, $N^{\circ} 71$. Venezuela (pp. 557-560)

Berk, Jonathan; DeMarzo, Peter; Harford, Jarrad (2010). Fundamentos de Finanzas

Corporativas. Pearson Educación, España

Blanch, Lluís; Elvira, Enric; Navalón, Marc (2001). Cash-management: Gestión de tesorería (segunda edición). Ediciones Gestión 2000, S.A., España.

Block, Stanley B.; Hirt, Geoffrey A.; Danielsen, Bartley R. (2013) Fundamentos de

Administración Financiera (decimocuarta edición). McGraw-Hill/ Interamericana Editores, México.

García-Aguilar, Juanita; Galarza-Torres, Sandra; Altamirano-Salazar, Aníbal (2017) Importancia de la administración eficiente del capital de trabajo en las Pymes. Ciencia Unemi. Volumen 10, $\mathrm{N}^{\circ}$ 23. Ecuador (pp. 30-39)

Gitman, Lawrence; Zutter, Chad (2012). Principios de Administración Financiera 
(decimosegunda edición). Pearson Educación, México.

Iturralde Jainaga, Txomin; Maseda García, Amaia; Ruiz de Agirre, Leire San José (2005). La gestión de la liquidez: necesidades y excedentes de tesorería. Boletín Económico de ICE. N 2834. España (pp. 29-41)

Keynes, John Maynard (1936). The general theory of employment, interest and money. Macmillan, Inglaterra.

Olsina, Xavier (2009). Cash Management. Revista de Contabilidad y Dirección. Volumen 9. España (pp. 63-78)

Promove Consultoría e Información SLNE (2012). Conceptos Básicos de Tesorería. C.E.E.I. Galicia, S.A. España.

Ross, Stephen; Westerfield, Randolph; Jordan, Bradford (2013). Fundamentals of Corporate Finance (novena edición). Tata McGraw-Hill Education, India

Sanz, Luis J. (2013). ¿Por qué debe un hospital tener una estrategia financiera? INCAE Business Review. Volumen 2, № 7. Costa Rica (pp. 26-32)

Terrazas Pastor, Rafael Alfredo (2009). Modelo de Gestión de Financiera para una Organización.

Perspectivas. № 23. Bolivia (pp. 55-72)

Torres, Adriana; Guerrero, Francisco; Paradas, Morella (2017). Financiamiento utilizado por las Pequeñas y Medianas Empresas Ferreteras. CICAG. Volumen 14, $\mathrm{N}^{\circ} 2$. Venezuela (pp. 284-303)

Van Horne, James; Wachowicz Jr, John (2010). Fundamentos de Administración Financiera (decimotercera edición). Pearson Educación, México. 